\title{
Les cellules souches hématopoïétiques : une population beaucoup plus hétérogène qu'il n'y paraît
}

Une série d'expériences publiées par Norman J. Wolf et al. (université de Washington, Seattle, USA) dans le numéro de mai 1993 d'Experimental Hematology apporte de très intéressantes informations confirmant la hiérarchie des cellules souches dans la moelle osscuse de souris [1].

Lc système hématopoiétique peut être présenté comme une pyramide au sommet de laquelle se trouve la population des cellules souches et dont la base est constituée par les cellules sanguines différenciécs. Entre ces deux extrêmes se positionne toute une cascade de progéniteurs hématopoïétiques qui, progressivement, se différencient dans les divers lignages hématopoḯtiques tout en perdant leurs capacités de prolifération $(\mathrm{m} / \mathrm{s}$ $n^{\circ} 6$, vol. 6, p.569, [2]). La notion de cellules souches est apparue rapidement, partant de la simple évidence que l'hématopoièse se maintient durant la vie de l'individu, avec un nombre considérable de cellules différenciées produites à chaque instant : nous disposons donc d'une population de cellules pouvant à la fois se différencier dans tous les lignages hématopoïétiques et s'automaintenir, deux propriétés qui servent à définir la cellule souche. La çonséquence de cette définition est que lorsqu'une cellule souche est transplantée dans un receveur conditionné, elle doit pouvoir repeupler tous les organes hématopoïétiques, myéloïdes et lymphoïdes, et assurer à l'infini la survie du receveur greffé.

Ce sont les travaux pionniers de Till et McCulloch à l'Ontario Cancer Institute (Toronto, Canada) en 1961 [3] qui semblèrent matérialiser l'existence des cellules souches hématopoïétiques. Il était connu que l'injection de cellules de moelle osseuse à un receveur $\mathrm{m} / \mathrm{s} n^{\circ} 8-9$ vol. 9, août-septembre 93 syngénique irradié le protège de la mort par déplétion hématopoïétique, ce qui, tout en confortant les caractéristiques fondamentales des cellules souches, ajoutait à leur potentiel celui de la transplantabilité [4]. Till et McCulloch constatèrent que l'injection d'un nombre réduit de cellules de moelle provoquait dans la rate des receveurs irradiés l'apparition en sept à huit jours de nodules hématopoïétiques, nodules fréquemment constitués de plusieurs lignages hématopoïétiques. Il fut démontré ultérieurement que ces colonies spléniques étaient d'origine clonale [5], qu'elles contenaient plusieurs lignages cellulaires [6] et que leur transplantation chez des receveurs secondaires donnait à nouveau des colonies spléniques [7]. Les cellules à l'origine de ces colonies spléniques (et dénommées pour cela colony forming unit in spleen; CFU-S) furent considérées pendant des années comme représentant la cellule souche, unique, multipotente et douée d'auto-renouvellement.

L'utilisation de substances qui détruisent les cellules en phase de synthèse de l'ADN (hydroxyurée, 5-fluorouracile,...) a permis de montrer que la majorité des cellules souches hématopoḯtiques sont quiescentes. Le maintien en G0 des cellules souches peut dépendre de la présence dans le micro-environnement de facteurs inhibiteurs [8, 9]. Ainsi, le tétrapeptide Ac-Ser-Asp-Cys-Pro peut empêcher l'entrée en phase S des CFU-S [10] tout comme la macrophage inflammatory protein-1a [11].

Par ailleurs, l'étude de la sensibilité des cellules souches hématopoïétiques à des agents cytotoxiques a apporté la première entorse à la théorie de la population de cellules souches uniques et multipotentes. Rosendaal et al. (Cancer Institute, Melbourne, Australia) [12] démontrèrent ainsi que les colonies spléniques obtenues après transplantation de moclle osseuse de souris traitées par l'hydroxyurée sont plus grosses et contiennent un taux de CFU-S plus élevé que celles obtenues à partir de moelle osseuse normale. Cette observation a conduit les auteurs à proposer une structure hiérarchisée de la population des cellules souches hématopoiétiques qui s'ordonneraient selon leur âge relatif, les plus immatures, hors cycle, ayant le plus fort potentiel de prolifération et d'autorenouvellement (generation age-hypothesis).

Ces travaux furent confirmés ultérieurement par Magli et al. au Basel Institut fïr Immunologie (Bâle, Suisse) [13] qui démontrèrent que les colonies spléniques observées au $8^{\mathrm{e}}$ jour n'étaient plus visibles au $12^{\mathrm{e}}-13^{\mathrm{e}}$ jour alors que pouvaient être observées des colonies spléniques non détectées au 8 e jour. Il fallait donc conclure à l'existence de deux populations de CFU-S qui furent définies sur la base des critères expérimentaux comme les CFU-S 8j et CFU-S 12j. Cette déf inition de l'hétérogéneité de la population des cellules souches se trouva confortée par les observations de Ploemacher et Brons à l'université Erasmus (Rotterdam, Pays-Bas) [14] montrant qu'il existe une population de cellules souches pré-CFU-S capables de produire les CFU-S 12j. Enfin la question semblait se compliquer encore quand il fut observé que des retransplantations systématiques de cellules médullaires d'un receveur à l'autre, espacées d'un mois, aboutissaient à la perte progressive des capacités de repeuplement [15], alors que des transferts plus espacés per- 


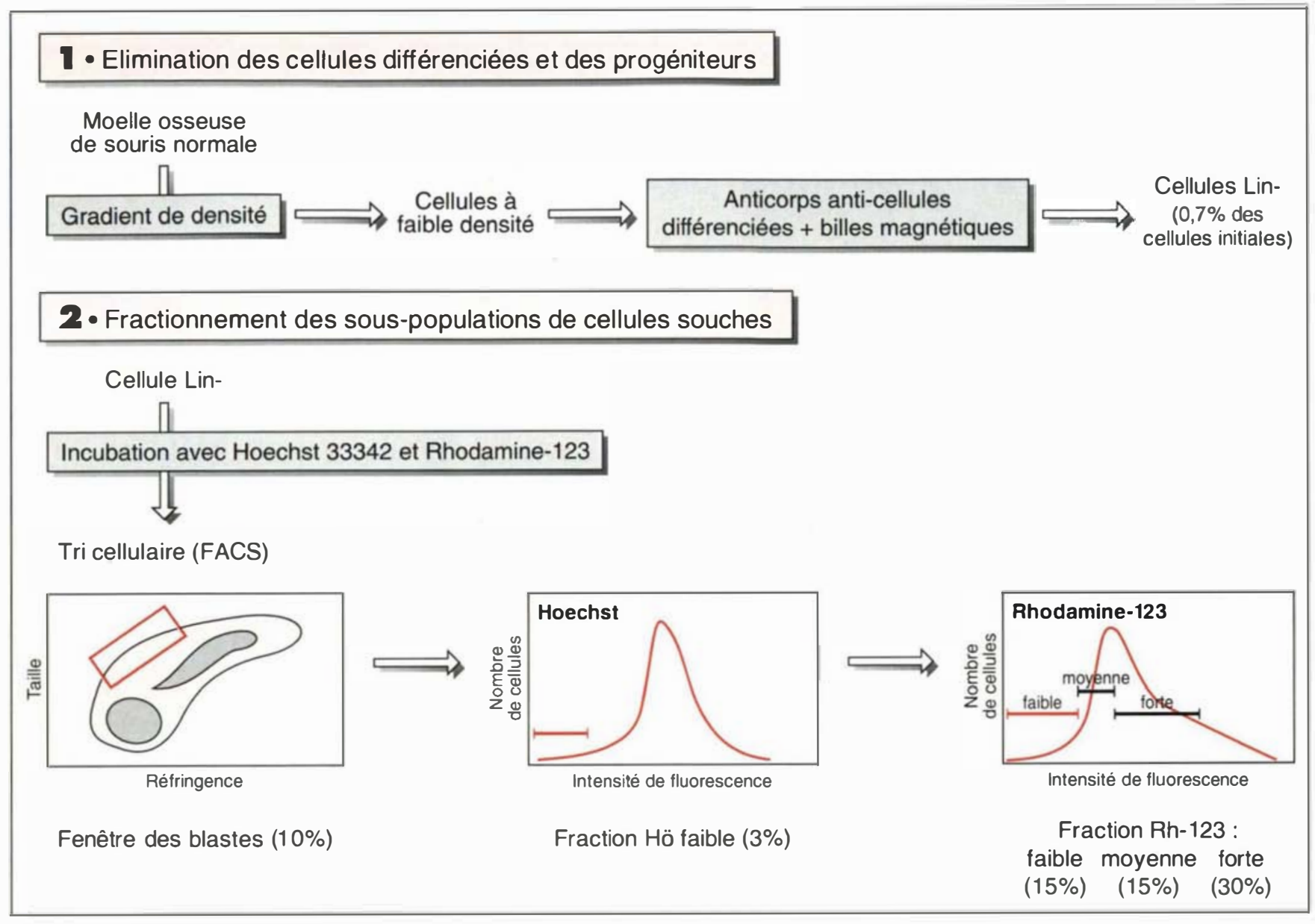

Figure 1. Schéma du protocole de fractionnement des populations de cellules souches utilisé par Wolf, et al [1]. FACS = fluorescence activated cell sorter.

mettaient le maintien des capacités de repeuplement [16].

Finalement, c'est le manque de définition expérimentale de la cellule souche hématopoḯtique qui entretient la confusion. Les auteurs s'accordent maintenant sur le fait que les CFU$\mathrm{S} 8 \mathrm{j}$ ou $12 \mathrm{j}$ ne sont pas les cellules souches les plus immatures. Cependant, nombre d'expérimentateurs utilisent comme critère expérimental, soit le test de reconstitution hématopoïétique à long terme chez des animaux irradiés, soit des essais clonaux révélant des cellules très immatures qui engendrent des colonies hématopoiétiques géantes (HPP-CFC ou highly proliferative potential-colony forming cell). reconnaissant des antigènes portés par les cellules souches et l'identification de colorants vitaux discriminant les cellules selon leur activité cyclique a permis à de nombreux groupes d'isoler, par cytofluorométrie, des fractions cellulaires très enrichies en cellules satisfaisant aux critères choisis des cellules souches. Pour une revue plus exhaustive, le lecteur peut se référer aux articles récents de Spangrude et al. (université de Stanford, CA, USA) [17] et Bartelemez et al. (Fred Hutchinson Cancer Research, Seattle, USA) [18].

Après avoir éliminé les lymphocytes $B$ et $T$, les macrophages, les granulocytes et les cellules de la lignée érythroïde à l'aide d'anticorps spécifiques, la purification peut consister en le tri des cellules exprimant faiblement Thyl et marquées positivement par un anticorps reconnaissant l'antigène Scal [19]. Vingt de ces cellules, enrichies de surcroît en cellules exprimant le recepteur c-kit du SCF (stem cell factor), peuvent repeupler aussi bien les lignages myéloïdes que lymphoïdes $B$ et $\mathrm{T}$ des souris irradiées [20].

Une autre possibilité réside dans le marquage des cellules Thy ${ }^{\text {low Lin- }}$ par le colorant Hoechst 33342 qui se fixe de façon réversible et vitale à l'ADN [21] ou la rhodamine 123 qui reconnaît les membranes mitochondriales [22]. L'expérience a prouvé que la sélection des cellules faiblement marquées par Hoechst 33342 [1] ou par la Rh123 [23] aboutissait à un enrichissement considérable en 
cellules capables de restaurer l'hématopoï̀se de souris irradiées. Bien évidemment de nombreuses expériences ont été rapportées qui croisent sélection antigénique et sélection tinctoriale, et aboutissent à un enrichissement considérable en cellules souches.

Le grand intérêt de l'étude récente de Wolf et al. [1] est d'avoir fractionné la moelle osseuse de souris selon une séquence de ces diverses méthodes et d'avoir alors étudié pour chaque fraction le taux de CFU-S $8 \mathrm{j}$ et $12 \mathrm{j}$, de HPP-CFC et de cellules reconstituant l'hématopoï̀se à long terme. Pour cela, les cellules de faible densité de la moelle de souris ont été d'abord recueillies sur gradient de densité, puis les cellules différenciées ont été éliminées par un cocktail d'anticorps, et les cellules blastiques sélectionnées par cytofluorométrie en fonction de leur taille et de leur complexité structurale (forward et right angle scatter); enfin, les cellules présentant une faible fluorescence après marquage par Hoechst 33342 (3\% des cellules) sont encore séparées en trois fractions selon l'intensité de la fluorescence après marquage par la rhodamine 123 en cellules faiblement, moyennement et fortement fluorescentes. Les différentes étapes de cet enrichissement sont représentées dans la figure 1. L'étude des potentialités de ces trois populations révèle des caractéristiques tout à fait intéressantes. Placées en culture in vitro en agar et en présence d'une combinaison d'IL3, IL1, CSF-1 et SCF, les cellules de ces trois fractions forment des colonies de type HPP-CFC de très grande taille avec une efficacité de repiquage ou de clonage de 50 à $100 \%$, pour les trois populations. Mais, lorsqu'est testée la capacité de ces cellules à former des colonies spléniques en 8 ou 12 jours chez des animaux irradiés, on constate alors que la population des CFU-S 8j disparaît rapidement des fractions les moins marquées par la rhodamine alors que les CFU-S $12 \mathrm{j}$ sont bien préservées. Lorsque l'on suit la survie des animaux à 30 jours, 200 des cellules des diverses fractions assurent une survie totale. Lorsqu'on réduit le nombre des cellules injectées apparaît un phé$m / s$ n० 8-9 vol. 9, août-septembre 93 nomène très caractéristique et déjà noté par d'autres auteurs : 150 cellules de la population Rh123 high préservent $70 \%$ des animaux alors qu'aucun receveur de 150 cellules Rh123 low ne survit. La différence entre les deux populations est donc criante. Comment expliquer ce phénomène? L'explication fournie est simple : les cellules souches Rh123 low, particulièrement immatures, ne peuvent assurer la prise de greffe immédiate (contrairement aux cellules souches $\mathrm{Rh} 123^{\text {high }}$ ), et les animaux meurent de déplétion hématopoïétique ou d'infection dans les jours qui suivent l'irradiation et la greffe. La vérification de cette explication tient dans l'expérience suivante, réalisée par les auteurs en utilisant la technologie mise au point par Szilvassy et al. (Terry Fox Laboratories, Vancouver, Canada) [24] : des femelles irradiées reçoivent 20 cellules de la fraction Rh123 low provenant de donneurs mâles et $3 \times 10^{6}$ cellules médullaires de femelles "altérées". L'altération consiste à passer chez deux receveurs successifs (secondaires et tertiaires) des cellules de moelle d'un donneur normal, l'intervalle de transfert étant de trois semaines à un mois. Cette opération a pour résultat la perte des cellules souches les plus immatures (qui n'ont pas le temps de rétablir leur nombre normal) et la préservation des progéniteurs les plus mûrs. Ces progéniteurs vont donc assurer dans les premières semaines la survie des animaux greffés, temps nécessaire à l'expression des cellules souches plus primitives, dont la descendance est évaluée par la présence du chromosome Y. Les résultats sont très clairs : 5 à 10 mois après la greffe, aussi bien les cellules de moelle des receveurs que leurs cellules $\mathrm{B}$ et $\mathrm{T}$ présentent à $100 \%$ le caryotype mâle. Quand la même expérience est réalisée avec les cellules Rh123 high, le caryotype mâle est faiblement représenté (et dans ce cas, pas dans les trois lignages), voire non détecté.

Ces expériences démontrent donc clairement que la population des cellules souches est hétérogène dans ses paramètres physiques mais surtout dans ses propriétés biologiques. Cer- taines peuvent assurer une reconstitution rapide après transplantation, d'autres ne le font que tardivement. La filiation des diverses cellules souches avec leurs principales caractéristiques est représentée dans la figure 2. La connaissance de la structure pyramidale de la population des cellules souches revêt un caractère fondamental chez l'homme: la manipulation des cellules à greffer ne doit pas aboutir à l'élimination de telle ou telle population au risque de perdre la capacité de ces cellules à assurer la reconstitution précoce et/ou tardive. Ces expériences démontrent également que le progéniteur de type HPP-CFC, dont la population est également hétérogène, est très voisin de, sinon identique à la cellule souche. Cela n'est pas sans intérêt dans la mesure où, pouvant être caractérisé in vitro, il peut être étudié facilement.

Cette hiérarchie fonctionnelle peut être déduite d'un autre type d'expériences qui ont apporté, de surcroît, des informations fort surprenantes sur le fonctionnement individuel des cellules souches. Les cellules souches peuvent être infectées, comme beaucoup d'autres cellules, par des rétrovirus défectueux pour la réplication et porteurs d'un marqueur génétique, en l'occurrence le gène bactérien néo qui confère la résistance à la néomycine. L'intégration de l'ADN viral (après transcription inverse) se réalise de façon aléatoire dans l'ADN cellulaire : chaque cellule souche infectée sera donc caractérisée par le site d'intégration de l'ADN viral, aisément reconnaissable par la taille des fragments de restriction auxquels le site d'intégration peut donner naissance. Il est donc possible de suivre à la trace les cellules provenant de telle ou telle cellule souche après greffe à un receveur irradié. On a pu ainsi démontrer une expression séquentielle de certains clones ; parfois même, un clone reste dormant chez le receveur primaire mais est détecté chez les receveurs secondaires [25]. Les quatre à six premiers mois qui suivent la greffe sont marqués par une fluctuation dans la prolifération des marqueurs, traduisant la mise en activité et l'arrêt de certaines cellu- 


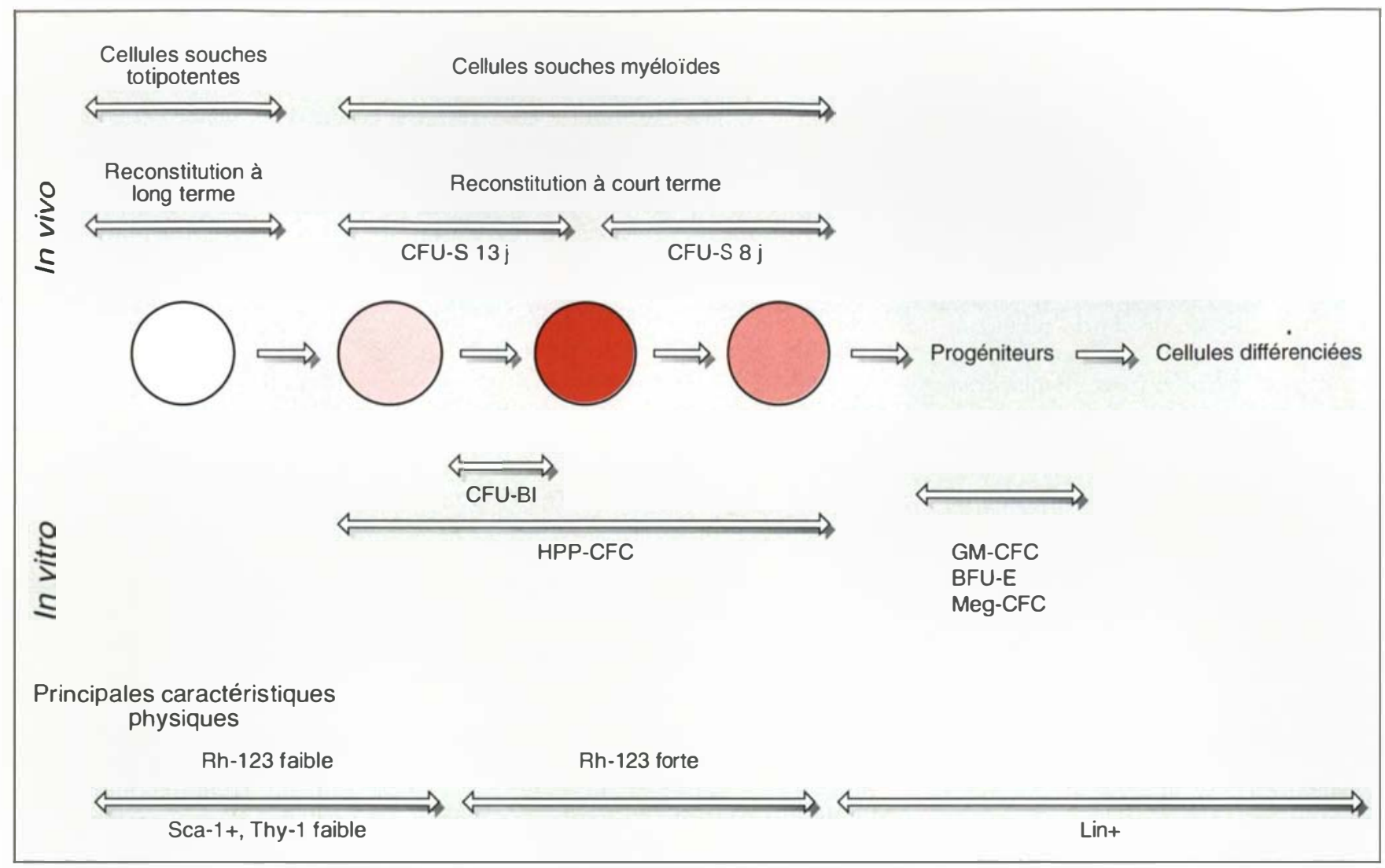

Figure 2. Filiation des différentes populations de cellules souches. Le schéma indique la capacité de ces cellules soit à reconstituer l'hématopoïèse de receveurs irradiés à court ou à long terme, soit à donner, in vitro, des colonies géantes de macrophages HPP-CFC (highly proliferative potential-colony forming cell), des colonies blastiques CFU-BI ou des colonies de cellules différenciées sous l'effet de facteurs de croissance appropriés. Figurent enfin les principales propriétés immunologiques et tinctoriales de ces diverses catégories cellulaires. CFU-S = colony forming unit in spleen. $G M-C F C=$ granulocyte-monocyte colony forming cell ; BFU-E = burst forming unit-erythroid; Meg-CFC = megacaryocyte-colony forming cell.

les souches. Graduellement, toutefois, un système stable s'établit, dominé par un petit nombre de cellules souches [26].

Dans un autre système, Van Zant et al. (Texas Tech University, Health Sciences Center, Lubbock, Texas, USA) [27] avaient observé que les souris DBA-2 ont une part importante de leurs CFU-S en cycle contrairement aux souris $\mathrm{C} 57 \mathrm{Bl} / 6$. Les populations sanguines de souris chimères $\mathrm{DBA}-2-\mathrm{C} 57 \mathrm{Bl} / 6$ sont initialement DBA-2 puis C57 Bl/6. La greffe de moelle allophénique* donne d'abord une hématopoïèse DBA/2 puis $\mathrm{C} 57 \mathrm{Bl} / 6$. La greffe de la moclle

- Allophénique : souris née de la fusion de deux blas. de ce receveur primairc à des receveurs secondaires montre alors la réapparition de l'hématopoï̀se de type DBA/2 qui, à nouveau, s'éteint avec le temps [28]. Là encore, toutes les cellules souches ne sont pas mises à contribution simultanément.

\section{Conclusion}

Les résultats rapportés ici permettent de jeter quelque lumière dans cette boîte noire qu'était, et est encore, la population des cellules souches hématopoïétiques.

Le concept qui prévaut aujourd'hui est que la population des cellules souches est organisée, hiérarchisée, comme l'est celle des progéniteurs.
Le sommet de la hiérarchie est occupé par une population de cellules dont on connaît quelques propriétés physiques et antigéniques : ces cellules souches sont apparemment responsables de la prise de greffe à long terme, mais ne peuvent assurer la prise de greffe immédiate. Ces propriétés sont raisonnablement en accord avec leur nature de cellules souches primitives. Il est donc fort probable que cette population soit " la vraie population " de cellules souches. Plus mûre, mais présentant naturellement les propriétés de cellules souches (autorenouvellement, pluripotentialité), se trouve une population de cellules souches assurant la prise de greffe immédiate. L'extinction de leur activité résulte-t-elle de leur épuise- 
ment par le bas de la population ou d'un mécanisme de rétro-contrôle se mettant en place lorsque se différencient (tardivement) les cellules souches issues de la cellule souche primaire ? La réponse ne peut être donnée pour le moment.

L'autre hypothèse est que toutes les cellules souches d'une population donnée ne sont pas en activité simultanément. Lorsque l'hématopoïèse a retrouvé son niveau d'équilibre, un faible nombre de cellules souches assurent la production hématopoïétique. Faut-il en conclure que la pérennité de la population des cellules souches résulte en fait d'une utilisation minimale de ses capacités de production et que le nombre des cellules souches est largement surdimensionné par rapport aux besoins, ou que cette pérennité est assurée par un autorenouvellement systématique de la cellule en division, l'une des cellules filles retournant à l'état initial ? Les expériences à venir devraient être très instructives dans ce domaine

\section{Jean-Paul Blanchet \\ Professeur}

Guy Mouchiroud

Chargé de recherche à l'Inserm

Université Claude-Bernard, Centre de génétique moléculaire et cellulaire, UMR 106, 43 boulevard du 11-Novembre-1918, 69622 Villeurbanne Cedex, France.

\section{RÉFÉRENCES}

1. Wolf NS, Kone A, Priestlez GV, Bartelmez $\mathrm{SH}$. In vivo and in vitro characterization of long term repopulating primitive hematopoietic cells isolated by sequential Hoechst 33342-rhodamine 123 FACS selection. Exp Hematol $1993 ; 21: 614-22$

2. Wendling F, Tambourin P. La superfamille des récepteurs de cytokines et l'oncogène v. mpl. medecine/sciences $1991 ; 7$ : 569-77.

3. Till JE, McCulloch EA. A direct measurement of the radiation sensitivity of normal bone marrow cells. Rad Res $1961 ; 14$ : 213-22.

$m / s$ n ${ }^{\circ} 8-9$ vol. 9, août-seplembre 93
4. Ford CE, Hamerton JL, Barnes DWH, Loutit JF. Cytological identification of radiation-chimacras. Nature 1956; 177 452-4

5. Wu AM, Till JE, Siminovitch L, Mc Culloch EA. A cytological study of the capacity for differentiation of normal hemopoietic colony forming cells. J Cell Physiol 1967 ; $69: 177-84$

6. Lewis JP, Trobaugh FC. Hæmatopoietic stem cclls. Nature 1964; 204: 589-90.

7. Curry JL, Trentin JJ, Wolf N. Hemopoictic splecn colony studies. II. Erythropoiesis. J Exp Med 1967; 125 : 703-20.

8. Frindel E, Guigon M. Inhibition of CFU entry into cyclc by a bone marrow extract Exp Hematol 1977 ; 5 : 74-6.

9. Lord BI, Mori KJ, Wright EG, Lajtha LG. An inhibitor of stem cell proliferation in normal bone marrow. $B r J$ Haematol 1976 ; $34: 441-3$.

10. Lenfant M, Wdieczak-Bakala J, Guit tet E, Prome JC, Sotty D, Frindel E. Inhibitor of hematopoietic pluripotent stem cell proliferation : purification and determination of its structure. Proc Natl Acad Si USA $1989 ; 86: 776-82$

11. Dunlop DJ,. Wright EG, Lorimore S Graham GJ, Holyoake T, Kerr DJ, Wolpe SD, Pragnell IB. Demonstration of stem cell inhibition and myeloprotective effects of SCI/rh MIP-1 $\alpha$ in vivo. Blood $1992 ; 79: 2221-5$

12. Rosendaal M, Hodgson GS, Bradlcy TR. Organization of hacmopoietic stem cclls : the generation age hypothesis. Cell Tissue Kinet 1976; 12:17-29.

13. Magli MC, Iscove NN, Ordatchenko $\mathrm{N}$. Transient nature of early hæmatopoietic spleen colonies. Nature 1982; 295 : 527-9

14. Ploemacher RE, Brons RHC. Separation of CFU-S from primitive cells reponsible for reconstitution of the bonc marrow hematopoietic stem cell compartment following irradiation. Evidence for a pre-CFUS compartment. Exp Hematol 1989; 17 : 263-6.

15. Hellman S, Botnick LE, Hannon EC, Vigneulle RM. Proliferative capacity of murine hematopoietic stem cells. Proc Natl Acad Sci USA 1978; 75 : 490-4.

16. Vos O. Multiplication of hemopoietic colony forming units (CFU) in mice after $\mathrm{X}$-irradiation and bone marrow transplantation. Cell Tissue Kinet 1972; 5 : 341-50.

17. Spangrude GJ, Smith L, Uchida N, Ikuta K, Heimf eld S, Friedman J, Weissman IL. Mouse hematopoietic stem cells. Blood 1991; 78 : 1395-402.
18. Bartelemez SH, Andrews RG, Bernstein ID. Uncovering the hetcrogeneity of hematopoietic repopulating cells. Exp Hematol $1991 ; 19: 861-2$.

19. Spangrude GJ, Heimfeld S, Weissman IL. Purification and characterization of mouse hematopoietic stem cells. Science $1988 ; 241: 58-62$

20. Ikuta K, Wcissman IL. Evidence that hematopoietic stem cells express mouse c-kit but do not depend on steel factor for their generation. Proc Natl Acad Sci USA 1992 89 : 1502-6.

21. Lalande ME, Ling V, Miller RG. Hocchst 33342 dyc uptake as a probe of membrane permeability changes in mammalian cclls. Proc Natl Acad Sci USA 1981; 78 363-67

22. James TW, Bohman R. Proliferation of mitochondria during the cell cycle of the human cell line (HL-60). J Cell Biol 1981 ; 89 : $256-60$

23. Bertoncello I, Hodgson GS, Bradley TS. Multiparameter analysis of transplantable hemopoietic stem cells. I. The separation and enrichment of stem cells homing to marrow and spleen on the basis of rhodamine 123 fluorescence. Exp Hematol $1985 ; 13: 999-1006$.

24. Szilvassy SJ, Fraser CC, Eaves CJ, Lansdorp PM, Eaves AC, Humphrics RK Retrovirus-mediated gene transfer to purified hemopoietic stem cells with long-term lympho-myelopoietic repopulating ability. Proc Natl Acad Sci USA 1989 ; 86 : 8798-802.

25. Lemischka IR, Raulct DH, Mulligan RC. Developmental potential and dynamic behavior of hematopoictic stem cells. Cell $1986 ; 45$ : 917-27.

26. Jordan CT, Lemischka IR. Clonal and systemic analysis of long-term hematopoiesis in the mousc. Genes Dev $1990 ; 4$ : 220-32.

27. Van Zant G, Eldridge PW, Behringer RR, Dewey MJ. Genetic control of hematopoietic kinetics revealed by analysis of allophenic mice and stem cell suicide. Cell $1983 ; 35: 639-45$.

28. Van Zant G, Scott-Micus K, Thompson BP, Fleischman RA, Perkins S. Stem cell quiescencc/activation is reversible by serial transplantation and is independent of stromal cell genotype in mouse aggregation chimeras. Exp Hematol $1992 ; 20$ : 470-5.

\section{TIRÉS A PART}

J.-P. Blanchet. 